\title{
Novel Multipoint Relays Scheme Based on Hybrid Cost Function
}

\author{
Ali Ouacha, ${ }^{1}$ Bachir Bouamoud, ${ }^{2}$ Ahmed Habbani, ${ }^{1,2}$ and Jamal El Abbadi ${ }^{1}$ \\ ${ }^{1}$ Laboratoire d'Electronique et de Communications (LEC), Ecole Mohammadia d'Ingénieurs (EMI), \\ Université Mohammed V-Agdal (UM5A), BP 765, Avenue Ibn Sina, Agdal, 10000 Rabat, Morocco \\ ${ }^{2}$ Laboratoire SIME, Ecole Nationale Supérieure d'Informatique et d'Analyse des Systèmes (ENSIAS), \\ Université Mohammed V-SOUISSI (UM5S), BP 713, Madinat Al Irfane, Avenue Mohammed ben Abdallah Regragui, \\ Agdal, 10000 Rabat, Morocco
}

Correspondence should be addressed to Bachir Bouamoud; bouamoud.bachir@gmail.com

Received 6 June 2013; Accepted 3 July 2013

Academic Editors: V. G. M. Annamdas, F. Lu, and Z. Mazur

Copyright (C) 2013 Ali Ouacha et al. This is an open access article distributed under the Creative Commons Attribution License, which permits unrestricted use, distribution, and reproduction in any medium, provided the original work is properly cited.

\begin{abstract}
When evaluating the performance of QoS protocols, a number of factors have a major impact on the results. Notably, QoS is emphasized when mobile ad hoc networks (MANETs) are employed into aerospace fields. Some of these parameters are a particular manifestation of characteristics of the MANET environment, such as mobility. Indeed, our proposal is a novel multipoint relays scheme based on hybrid cost function taking into account QoS criteria and avoiding mobility effect of nodes, especially those selected as MPRs. A comprehensive simulation study was conducted to evaluate the performance of the proposed scheme. Performance results show that RQMPR outperforms existing MPR heuristic adopted in the ad hoc routing protocols OLSR and QOLSR, in terms of packet delivery and average end-to-end delay.
\end{abstract}

\section{Introduction}

Ad hoc network is wireless network composed of autonomous individual nodes. It is easy to install and deploy and provides point to point communications between nodes without any infrastructure network. In an ad hoc network, since there is no central coordinator, for example, an access point (AP) or base station (BS), all nodes are supposed to work as terminals and routers at the same time. Thus, a routing protocol will play a major role in an ad hoc network to connect nodes that cannot communicate with each other directly and does not stop to be a subject of research work to improve the performance of wireless networking solutions.

Due to the dynamic changes of the factors that affect the performance in a mobile ad hoc network, it would be convenient that any proposed optimizations should consider the dynamics that act on nodes and links which interconnect them. In this context, knowledge of network must also have the same character in terms of taking into account factors such as available bandwidth, delays, and the lifetime of nodes in the process of selection of multi point relays.

In proactive routing mechanism, the use of relays aims to reduce the broadcast messages senders and then the number of flooded messages; here we highlight the importance of relays in the OLSR protocol case since they become the only responsible for broadcasting Topology control messages. While this approach is pleasing to the eye, beside some control functions that are necessary to prevent an eternal duplication of broadcast messages, it is required to selected relays in a reliable manner; indeed, defects in the reception of broadcast packets from MPR nodes can greatly affect the rate of delivery of packets across the network.

QoS routing relies on the state of parameters specifying resource availability at network nodes or links and uses them to find paths with enough free resources, and this concept can be applied also on the selection of relays, by choosing among the candidates nodes those maximizing a composite constraint based on the path characteristics to reach a 2-hop farther nodes.

In Figure 1, we present a case that highlights the process of MPR selection in node $V_{1}$, where $V_{2}$ is the only intermediate node to reach $V_{5}$. Besides $V_{6}$ and $V_{7}$ are reachable through both nodes $V_{3}$ and $V_{4}$; thus, $V_{1}$ must make a decision to select which one of them to be in its MPR set. Although in the standard OLSR (RFC 3626) the selection is made randomly 


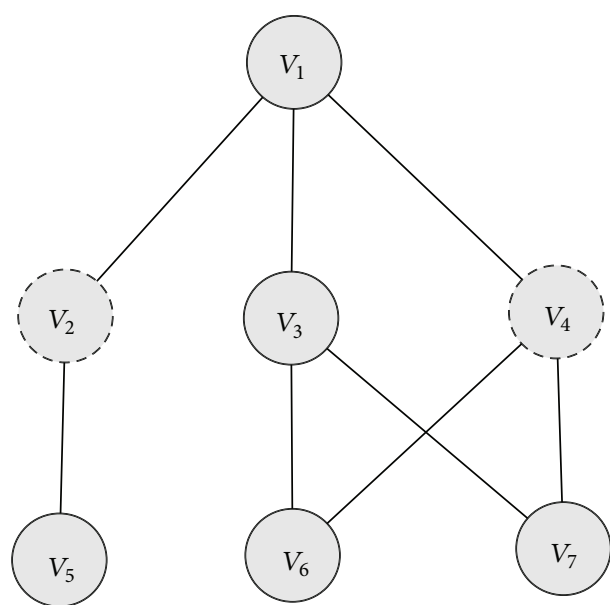

FIgURE 1: MPR selection case study.

among all the candidates, node is to be selected as a relay. Related to this situation, the question is how can we avoid a random choice and select the most convenient node between $V_{3}$ and $V_{4}$ ?

In the rest of the paper, we refer to our proposal as RQMPR, and it is organized as follows. In Section 2, we present some previous works whose aim is to improve routing reliability. In Section 3, we introduce a new composite metric. In Section 4, we present a new MPR selection heuristic. Section 5, contains a set of simulations and results which concern the evaluation of our approach. In Section 6, we evaluate the performance of the proposed scheme. Lastly in Section 7, we conclude and discuss future works.

\section{Related Work}

Multipoint relay nodes publish inside the TC messages the links which could constitute the paths from source to destination; then the set of MPRs form a kind of backbone, in the mobile ad hoc network. Thus, one promising issue of the routes selection optimization is to carefully select MPR that meets a given requirement to improve the targeted network performance. Indeed, an analysis of MPR selection in the OLSR Protocol [1] concluded that routes performance can be increased by adopting some supplementary criteria on MPR mechanism. In other words, the routing metrics can be also taken into consideration to choose the nodes relays, to face up to the nonadvertisement of potential link [2]. Most of the literature of routing optimization in OLSR aims to find other efficient metrics rather than the default one defined in the RFC3626, where the path quality is measured by the number of hops.

Some proposed routing metrics are based on Mac layer information such as the queue length, bandwidth, or Bit Error Rate (BER) [3]. QOLSR [4] is one of the major QoS extension in the proactive routing category. It enforces restrictions on bandwidth availability and/or delay by evaluating these metrics using a heuristic method that provides an option of best effort routing by searching for a so-called shortest-widest path. However, a mean drawback of this approach is that the number of generated MPRs is increased since the criteria of available bandwidth are a prior condition to reachability for choosing a node as a relay indeed, and this situation causes control overhead due to excess of topology control messages, particularly in a very mobile context where links are broken frequently, so it is not efficient to select MPR whose links that provide a high QoS, but it is lost in few coming moments.

MPRs utilization decreases the number of retransmissions to disseminate a broadcast packet, and a relay selection method has been proposed in [5] to further reduce retransmissions by aggregating broadcast packets at MPR even if reducing the number of retransmissions may not be necessarily advantageous for reliable packet delivery due to instability of radio support. A reverse approach has been proposed in [6] based on choosing a redundant MPR coverage for relays in 2-hop neighbourhood of a given node in this manner; routing overhead is generated as consequence.

In cross-layer measurement, an early approach called signal stability routing in [7] consists of prioritizing the paths whose links have the strongest signal. In a similar way, authors present preemptive routing techniques in [8] to calculate approximately the time of link breakage so that failure can be announced in advance. Moreover, ETX (expected transmission count) metric [9] has been proposed as an MANET internet draft, and it is in the way to become a standard. Thereby in [10], a comparison work has been done among OLSR and RFC with default hop count metric and OLSR-ETX metric in a mesh network. Their results reveal the ETX metric to be fundamentally imperfect when assessing optimal routes in large dense mesh network scenario and worse than the OLSR-RFC standard. Despite of that in the various literature [11-13], a different approach is adopted based on seeking the well-suited parameter configurations of existing mobile ad hoc network protocols in order to get better performances. In the same way, others [14] propose a cross-layer design that jointly considers routing and topology control and take mobility and interference into account.

Nevertheless, many works discuss routing optimization based on online nodes measurements in order to categorize paths which are preferentially used for routing. But these works have a common weakness, where they cannot prevent possible change in links status occurring in the future. A link qualified as reliable based on past or current measurements may become unreliable with time because of dynamic nature of mobile environments.

In some works, authors draw attention to the stability of routes; indeed, in [15], the objective is to seek for stable paths between source and destination that also have lower hop count using the "predicted link expiration time (LET)" concept [16] employed for the Flow-oriented routing protocol (FORP) [17]. The FORP has been observed to look for stable routes that have even twice the highest lifetime and the minimum-hop routes discovered using the well-known dynamic source routing (DSR) protocol [18]. However, the trade off is that the hop count of FORP routes is significantly larger (twice or more) than the minimum hop count [19].

So far, many foregoing studies focus on statistical examination of link availability. The study made in [20] showed 


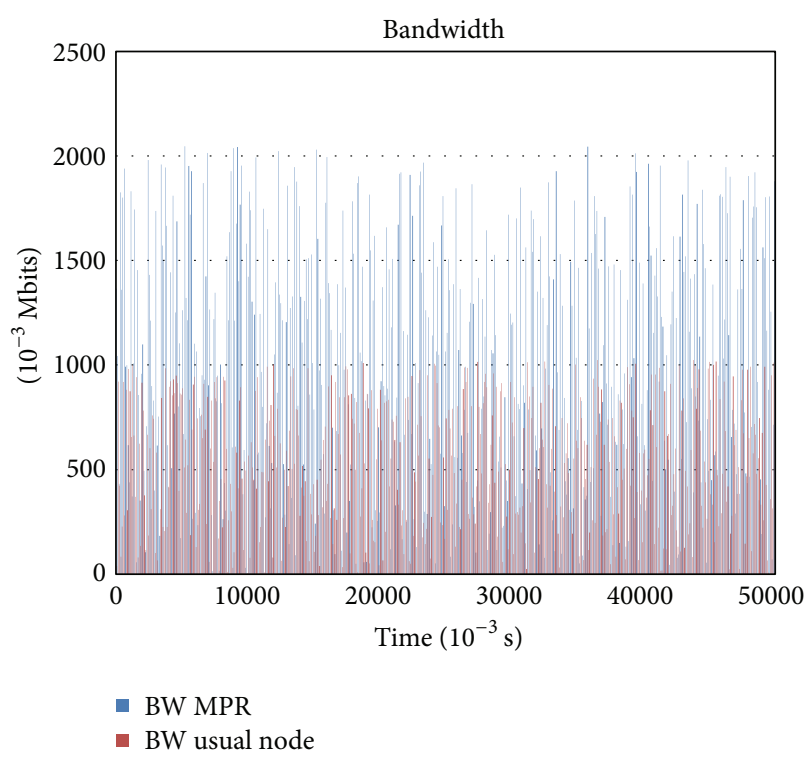

FIgURE 2: Bandwidth consumption.

that the link lifetime has a multimodel distribution when the node's speed is slow, and the path availability duration can be approximated by an exponential distribution at moderate and high velocities. On the other hand, the solutions provided by these studies are valid only for some specific situations; they could not be completely extended to universal ad hoc networks and practical MANETs applications. In [21, 22], a prediction method has been proposed and investigated with random walk mobility model based on link availability estimation. The algorithm aims to predict the probability of a link available with a continuously manner for a certain period, which is obtained based on the current node's movement. However, this algorithm is inaccurate when calculating the link availability; it makes the tendency of a given link availability known. Additionally, another approach [23] tries to predict the link availability during a time span based on a rough estimation of the distance between two nodes.

Routes rediscovering causes significant data loss, communication overheads especially with reactive routing behaviour, and jitter, so, routing protocol for mobile ad hoc networks must match the mobility character. In this way, our reflexion aims to adopt a composite metric taking into account the online measurement such as bandwidth/delay and links lifetime in the selection of relays.

Different mobility models can be used to evaluate MANET routing protocols performance. They can be classified into two categories: entity and group mobility models. Detailed reviews of these models can be found in [24-26]. In this paper, we will use random waypoint (RWP) [27].

\section{Background}

Metrics must accurately capture the triggered link characteristic, based on direct measurement or even on prediction computing. An obvious approach is to express the resource availability at a given node by a unique measure (total cost) and then use it as a single metric. For example, we can express the metrics of the bandwidth and the delay of a path by a function proportional to the bandwidth and inversely proportional the delay. However, this approach does not take into account the possible change in future time.

\subsection{Cross-Layer Measurement}

3.1.1. Bandwidth. Bandwidth metrics are popular, especially for QoS applications. Indeed, it indicates the capacity of data which can be sent through a link within a time spanner. From the perspective of a node, this is equal to the transfer rate of a link. Many factors other than theoretical physical bandwidth have a significant effect on this metric such as packet loss ratio. It Even refers to the measurement of bandwidth that is reduced to the determination of the number of free communication slots.

Firstly, we highlight the fact that nodes selected as MPRs introduce more traffic (context of OLSR). Indeed they generate TC messages on which links with the MPR selector set are published by relay. Beside that, Figure 2 presents bandwidth consumption by MPR, within simulation environment according to Table 2.

Nonetheless, bandwidth graph reveal; the debit consumed afterwards simulation but to make decision the mechanism of the latter must instantaneously give the available bandwidth.

Definition 1 (capacity of link). For a pair of nodes within transmission range of each other, we define the capacity $C$ of the link between them as the physical transmission bit rate of the source node.

Definition 2 (bandwidth of link). In absence of competing stations, the time to get and release the medium in a onehop transmission is a random variable $T$. The time required to transmit an $L$-bit long packet at a link transmission rate of $C$ bps will be $T+L / C$, which means that if the link is completely available as follows the link bandwidth is

$$
\mathrm{BW}=\frac{L}{T+L / C} \text {. }
$$

In fact, $L / C$ quantity represents the physical transmission time and $T$ represents, fading, internal, external noise, and contention; let us aggregate the formula (1) and express it in 802.11 way, considering the packet size and the time spanner between sending and acknowledgement,

$$
\mathrm{BW}=\frac{L}{t_{q}+\left(t_{S}+t_{\mathrm{CA}}+t_{\mathrm{Over}}\right) \times R+\sum_{r=1}^{R} B_{\mathrm{off}}^{r}},
$$

where $t_{q}$ is the Mac layer queuing time, $t_{S}$ is the transmission time of the $L$ bits, $t_{\mathrm{CA}}$ is the collision avoidance phase time, $t_{\text {Over }}$ is the control overhead time such as ACK and RTS/CTS, $R$ is the necessary retransmissions, and $B_{\text {off }}^{r}$ is the back-off time for a retransmission $r$.

This formula reveals some undesirable characteristics that are however common to measurements such as packet size dependence. Thereby, in our calculation, the BW is averaged 


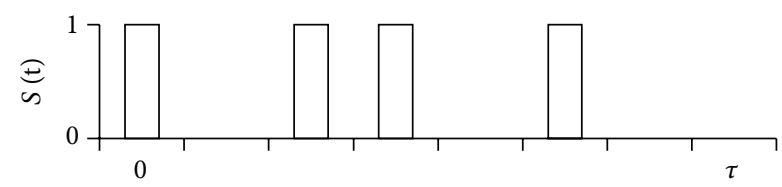

FIGURE 3: Link utilisation during a time span estimation.

TABLE 1: Cost comparison of QoS-based MPR schemes.

\begin{tabular}{ccccc}
\hline Schemes & I.R & S.D & T.C & M.C \\
\hline RQMPR & 2 hops & Yes & $O\left(2\left|N_{1}\right|\left|N_{2}\right|\right)$ & $O\left(\left|N_{1}\right|+\left|N_{2}\right|\right)$ \\
QMPR-1 & 2 hops & Yes & $O\left(3\left|N_{1}\right| \sigma+\left|N_{2}\right|\right)$ & $O\left(\left|N_{1}\right|+\left|N_{2}\right|\right)$ \\
QMPR-2 & 2 hops & Yes & $O\left(3\left|N_{1}\right| \sigma+\left|N_{2}\right|\right)$ & $O\left(\left|N_{1}\right| \sigma+\left|N_{2}\right|\right)$ \\
QMPR-3 & 2 hops & Yes & $O\left(2\left|N_{1}\right|\left|N_{2}\right|\right)$ & $O\left(\left|N_{1}\right| \sigma+\left|N_{2}\right|\right)$ \\
\hline
\end{tabular}

$\left|N_{1}\right|$ : the maximum number of 1-hop neighbours of a node. $\left|N_{2}\right|$ : the maximum number of 2-hop neighbours of a node. $\sigma$ : the maximum MPR number of a node.

TABLE 2: Simulation parameters.

\begin{tabular}{lc}
\hline Simulation environment & Option and parameter \\
\hline Flat size & $1000 \mathrm{~m} \times 1000 \mathrm{~m}$ \\
Max number of nodes & 70 nodes \\
Radio scoop & $100 \mathrm{~m}$ \\
MAC layer & IEEE.802.11.peer to peer mode \\
Transport layer & User datagram protocol (UDP) \\
Traffic model used & CBR \\
Package size & 512 bytes \\
Rate & 0.4 \\
The number of connections & $1 / 5$ of the number of nodes \\
Mobility model & Random waypoint (RWP) \\
Pause time & 0 second \\
Maximum speed of nodes & $5,10,15,20$, and $30 \mathrm{~m} / \mathrm{s}$ \\
Simulation time & $200 \mathrm{sec}$ \\
\hline
\end{tabular}

according to past values, which mitigates the foregoing dependences.

Definition 3 (available bandwidth (ABW)). It is defined as the unused bandwidth over the time interval $\tau$. Here $\tau$ is referred to as the estimation period (namely, the time needed for estimating $\mathrm{ABW}$ once). It is not a constant value and can be changed in different estimation tools, or even in a tool according to the network scenario.

The state of a given link $i$ is at time $t$ is as follows:

$$
S_{i}(t)= \begin{cases}0, & \text { link is idle } \\ 1, & \text { link is busy. }\end{cases}
$$

As it is shown in Figure 3, then the average utilisation of a link is as follows:

$$
\bar{u}_{i}=\frac{1}{\tau} \int_{t-\tau}^{t} S_{i}(t) d t
$$

The available bandwidth in a time interval of $[t-\tau, t]$ is as follows:

$$
\mathrm{ABW}_{i}=\left(1-\bar{u}_{i}\right) \mathrm{BW}_{i} .
$$

In the rest of paper, the available bandwidth is referred to as bandwidth.

3.1.2. Delay. The average end-to-end delay is the average time taken between packet sending and successful message receiving from the source to the destination. Similar to the QOLSR [4], each node includes in the Hello message the creation time of this message. When a neighbour node receives this message, it calculates the difference between such time and the current time; this is done in a synchronized network. Due to the characteristics of sparse ad hoc networks, classical clock synchronization algorithms are not applicable. For the moment, the aim of the current work is not to answer synchronization issues. Time synchronization in ad hoc networks is a wide subject of research, such as the work presented in [28]. This metric is important in delay sensitive applications such as video and voice transmission:

$$
\begin{aligned}
& \text { Average End to End Delay } \\
& \qquad=\frac{\sum \text { received_Time }- \text { sent_Time }}{\text { Total_Data_packets_received }} .
\end{aligned}
$$

3.2. Remaining Time to Quite (RTTQ). In a previous work, [29] we have introduced a new metric called RTTQ remaining time to quite which estimates the remaining time of each node to be unreachable by the node executing the MPR selection process, based on the distance and the radio scoop for a given speed. We assume that between each pair of nodes, there is only one link, and for a given node, the remaining lifetime of the peer node is equivalent to the link lifetime between them. Here we talk about prediction because of the prior idea that we get, by knowing when the link status is susceptible to being invalid.

The Remaining time to quite (RTTQ) (Figure 4) of each neighbour node referred to as $V_{\text {peer }}$ (peer node) is susceptible to leaving the neighbourhood of a reference node $V_{\text {ref }}$; running MPRs selection procedure is estimated based on travelled distances at two consecutive messages receptions (positions $P_{t}$ and $P_{t+\tau}$ ). These positions are computed based on nodes abscises and axis coordinates and the elapsed time during this travel $\llbracket t, t+d t \rrbracket$ and radio scoop (RANGE), where the instants $t$ and $t+d t$ are the time duration to reach the positions $P_{t}$ and $P_{t+\tau}$, respectively. Indeed, the sign of $\Delta_{t, t+\tau}$ gives an idea about the direction of each neighbouring node relatively. So the positive value $\left(\Delta_{t, t+\tau} \geq 0\right)$ indicates that distance between the pair of nodes is getting larger and a negative value $\left(\Delta_{t, t+\tau}<0\right)$ indicates they are closer. Indeed, in this case, link failure is unlikely; thus, RTTQ is set to its maximum value. However, in the opposite case, we predict the moment of the connection loss. Then, in time interval $\llbracket t, t+d t \rrbracket$, we calculate the travelled distance relative speed $\vartheta(7)$ the node $V_{\text {peer }}$ (assuming the $V_{\text {reef }}$ node as a reference) 
to reach the radio scoop edge. The estimated RTTQ is given by (9) as follows:

$$
\begin{gathered}
\Delta_{t, t+\tau}=D_{t+\tau}-D_{t}, \\
\vartheta=\frac{\Delta_{t, t+\tau}}{\tau}, \\
\operatorname{RTTQ}(t+d t)=\frac{\operatorname{RANGE}-D_{t+\tau}}{\vartheta} .
\end{gathered}
$$

3.3. Network Model. We assume that a network has been a direct graph $G(V, E)$, where $V$ is the set nodes and $E$ the set of links $l=(x, y)$ where the node $y$ is within the transmission range of $x$. In general cases, routing metrics have an attributed values which we call weighs associated to each edge. Consider

$$
\begin{aligned}
& \operatorname{dist}(x, y) \text { : number of hops between node } x \text { and node } \\
& y \\
& N_{1}(x)=\{y \mid \operatorname{dist}(x, y)=1\} \text {, set of 1-hop adjacent } \\
& \text { nodes } \\
& N_{2}(x)=\{y \mid \operatorname{dist}(x, y)=2\} \text {, set of 2-hop adjacent } \\
& \text { nodes. }
\end{aligned}
$$

Definition. Consider the following.

$B_{2}(x)$ is bandwidth of 2-hop farther path; it is a concave metric equal to the minimum of link bandwidth composing the path to a given node in $N_{2}(x)$.

$\operatorname{Del}_{2}(x)$ is delay of 2-hop farther path; it is an additive metric equal to the delay sum of intermediate links.

$\mathrm{RTTQ}_{c}(x)$ is the remaining time to quite for a node in $N_{1}(x)$, by which node $x$ reaches a given node in $N_{2}(x)$.

$F_{i}(x)$ is the function cost of each available $i$ path between source $x$ and $y$ node in $N_{2}(x)$, in which we aggregate three objectives by maximizing bandwidth and RTTQ and minimizing the delay, where

$$
F_{i}(x)=B_{2}(x)+\mathrm{RTTQ}_{c}(x)-\operatorname{Del}_{2}(x) .
$$

For accuracy care, the cost function is achieved by a normalized weighted additive utility function. Indeed, each metric is as follows:

$$
\begin{aligned}
B_{2}(x) & =\frac{b_{2}(x)}{\max _{x \in V} b_{2}(x)}, \\
\operatorname{del}_{2}(x) & =\frac{\operatorname{del}_{2}(x)}{\max _{x \in V} \operatorname{del}_{2}(x)}, \\
\mathrm{RTTQ}_{c}(x) & =\frac{\mathrm{RTTQ}_{c}(x)}{\max _{x \in V} \mathrm{RTTQ}_{c}(x)},
\end{aligned}
$$

where $b_{2}(x)$ is measured bandwidth, $\operatorname{del}_{2}(x)$ is measured delay, and $\mathrm{RTTQ}_{c}(x)$ is measured RTTQ.

\section{Proposal Heuristic}

In general, all the MPR broadcast schemes based on crosslayer information aim to revise the original MPR selection heuristic to achieve QoS awareness. However, it also has more relays with other MPR schemes, therefore growing retransmissions in the networks.
In [30] two schemes based on QoS measurement are proposed. First heuristic referred to as QMPR-1 still has the same initialization steps as the original MPR heuristic, except it changes procedure in order to provide QoS priorities. Instead of higher degree, a node with maximum bandwidth is chosen in case of multiple choices. If equal solution still exists, a node with minimum delay is selected. Then, likely MPRs with large bandwidth are selected, but the improvement is insignificant. Second heuristic, referred to as QMPR-2, is similar to the first one but selects nodes with higher bandwidth as MPRs, and the delay is used when there is a tie. And in case of multiple node with maximum delay reachability is choises. This heuristic highlights QoS criteria in the MPR selection; thus, MPRs are chosen based on QoS conditions, so the optimal links are published between a given pair of source and destination. Another heuristic referred to as QMPR-3 has been proposed in [31] and surveyed by [32], based on the idea that lets all 2-hop neighbours have an optimal bandwidth path through MPRs to the source node. For each 2-hop neighbours $y, a$ node $x$ chooses from its 1hop neighbour node as the MPR if it covers $y$, and the path is the largest from $y$ to $x$. Each 2-hop node has to go through this calculation until it finds an optimal path to the source node.

A survey [32] has established a comparative QoS-based MPR heuristics such as QMPR-1, QMPR-2, and QMPR-3 based on the following.

Time Complexity (TC). The maximum number of steps required in the worst case of a heuristic.

Message Complexity (MC). The maximum number of messages used in the worst case for a heuristic to obtain necessary information.

Information Range (IR). The number of hops of neighbor's information is required.

Source Dependent (SD). Before forwarding, node needs to know whether or not messages it received are from its MPR selectors.

Beside that in Table 1, we extendc this comparison work by including our proposal MPR schemes referred to as RQMPR based on a cost function. It still follows the same steps as the original MPR heuristic but modifies priorities action done in step (4) (Algorithm 1) where, for each 2hop node, the cost function $F_{i}(x)$ of all of its available paths to the source node and reachability of candidate relay are calculated. This step takes $O\left(\left|N_{1}\right|\right)$ time to be complete in the worst case when a 2-hop node is reachable by all onehop nodes. Then, for each two-hop node, add to the MPR set a node that can provide the maximum cost function. This step takes $O\left(\left|N_{1}\right|\right)$ time to run. Since these two steps have to be operated for all two-hop neighbours, the total time complexity of the heuristic can be $O\left(2\left|N_{2}\right|\left|N_{1}\right|\right)$. Our proposal is source dependent because it need information to be included in broadcast messages in order to decide whether or not a node will relay the message. Furthermore, it requires the information range of 2 hops; thereby, the message complexity is $O\left(\left|N_{2}\right|+\left|N_{1}\right|\right)$. 
Initial: A node $k, N_{1}(k), N_{2}(k)$.

Return: $\mathrm{MPR}_{k}$; MPR set of $k$

begin

(1) Add to $\mathrm{MPR}_{k}$ the node in $N_{1}(k)$ which is the only one to reach a node in $\mathrm{N}_{2}(k)$

(2) Remove the nodes from $N_{2}(k)$ which are covered by a node in $\mathrm{MPR}_{k}$

(3) While $\left(N_{2}(k)\right.$ not empty) do For each node in $N_{1}(k)$,

(i) Calculate the number of nodes in $N_{2}(k)$ that it can reach, that is, reachability

(4)

(ii) Calculate $F_{i}(k)$ to reach $N_{2}(k)$ Add to $\mathrm{MPR}_{k}$ the nodes which has the highest $F_{i}(k)$, If multiple choices, add which provide the highest reachability. If multiple choices, select node with highest degree, that is, number of 1-hop neighbors.

(5) Remove the nodes from $\mathrm{N}_{2}(k)$ which are covered by a node in $\mathrm{MPR}_{k}$

end

Algorithm 1: RQMPR broadcast schema (RQMPR selection).

\section{Simulation Environments}

Simulations are done in NS2 [33] (network simulator) version 2.35 in which we have integrated a standard version of OLSR. (UM-OLSR-0.8.8 [34, 35]), which is developed by MANET Simulation and Implementation at the University of Murcia (MASIMUM).

Our simulation parameters are as follows: for all simulations, our network consists of a maximum numbers of mobile nodes (70) whose radio scoop is $100 \mathrm{~m}$, moving in an area of $1000 \times 1000 \mathrm{~m}^{2}$. Each node moves according to the random waypoint (RWP) mobility model [36] with pause time fixed to 0 second and maximum speed varying between 5 and 30 meter/second with step (5). The scenario that defines the nodes movement is regenerated at the beginning of each simulation. To generate traffic in the network, in each simulation, 1/5 of nodes are randomly selected to be a source of constant bit rate (CBR) traffic. And these selected nodes use user datagram protocol (UDP) connections to send Packets with 512 bytes of size in the order of one packet every 2.5 seconds. Table 2 summarizes all the parameters used during simulations.

Within the simulations set, we distinguish all the possible attributes of the network, such as node mobility and the node density according to the following.

Varying Number of Nodes. We vary the number of nodes in the network. Our objective is to investigate the impact of node density on the protocol's performance. We use the same simulation area as in our previous simulations and gradually increase the number of nodes in the network. A desirable property of a protocol is to have stable behavior regardless of the number of nodes in the network. However, due to wireless medium limitations, we do not place an inadequate number of nodes in the simulation area. A small number of nodes in a large simulation area will result in low connectivity due to the large distances between nodes. In contrast, a large

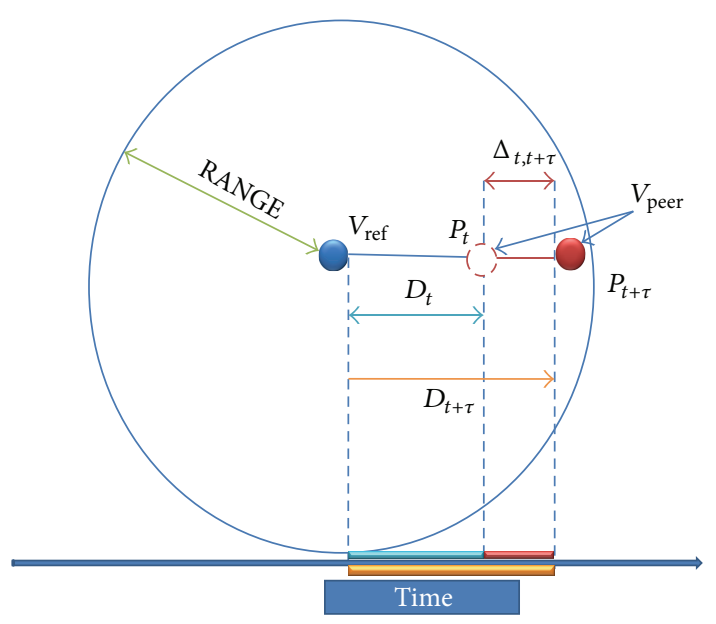

FIGURE 4: Comprehensive case of RTTQ computing.

number of nodes in a small simulation area will result in signal interference, as nodes are located very close to each other.

Varying Network Mobility. We vary the mobility of nodes. We start with a mobility scenario in which the nodes have a low velocity of $5 \mathrm{~m} / \mathrm{s}(18 \mathrm{~km} / \mathrm{h})$. We then increase the node velocity up to $30 \mathrm{~m} / \mathrm{s}(108 \mathrm{~km} / \mathrm{h})$. Our intention is to investigate the behaviour of protocols in networks with varied mobility although the high mobility $(108 \mathrm{~km} / \mathrm{h})$ cannot be easily found in military movements. We observed that, at higher data rates with increasing mobility, the performance of the protocols decreases. The decreased performance is large, mainly due to network congestion, in a way that makes any comparison meaningless.

All the metrics of performances should be based on same network attributes, such as mobility, network density, data density, bandwidth, energy resources, transmission and 


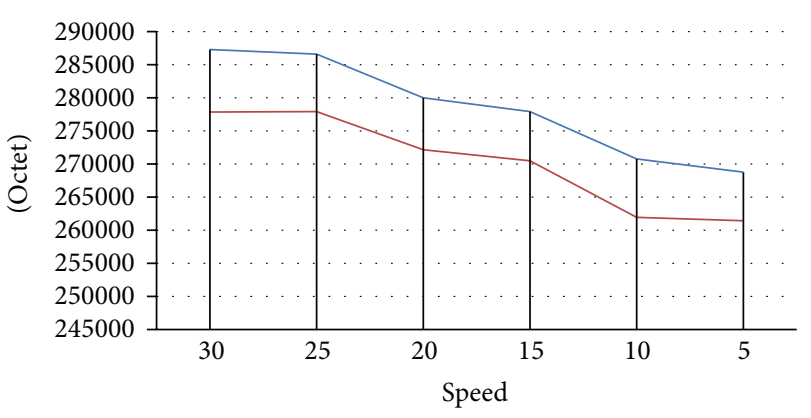

— Average MPR ABW

— Average neighbour ABW

FIgURE 5: Average ABW for neighbours and MPRs.

receiving power, antenna types, and any other "component" that affects the performance of a routing protocol. In our performance evaluation, we follow the general ideas described in RFC 2501 [37] and use some quantitative metrics to evaluate our proposal RQMPR using as cost function $F_{i}(x)$, the standard one in OLSR (MPR-1), and QOLSR referred to as QMPR-2.

\section{Evaluation of Performance}

Previously, we have highlighted the need of bandwidth nodes selected as MPRs in OLSR where the link attributes are not considered in the selection of those relays. In this step, we check the effect of our proposal to deal with that problem in Figure 5.

Indeed, it reveals that the selected MPRs have more available bandwidths, and it scales well for different velocities.

6.1. Packet Delivery Ratio. The packet delivery ratio is defined as the fraction of all the received data packets at the destinations over the number of data packets sent by the sources (14). This is an important metric in networks. Indeed, packet loss at the intermediate nodes will result in retransmissions by the sources, which may result in network congestion,

$$
\text { Packet Delivery Ratio }=\frac{\text { Total Data packets received }}{\text { Total Data packets sent }} \text {. }
$$

6.2. Throughput. It indicates the capacity of data which can be sent over the network within a given time. From the perspective of a node, this is equal to the transfer rate of a link. Many factors other than theoretical physical bandwidth have a significant effect on this metric, for example, packet loss ratio.

Figure 6(a) presents the packet delivery ratio for both protocols MPR-1 and QMPR-2, and we see that it deteriorates while speed is getting higher. Despite that, QMPR-2 dominates MPR-1 by almost 15\% of more successfully delivered packets.
Figure 6(b) presents the latter measure of performance for both protocols in different densities, where there is no meaningful difference until the number of nodes reaches 60 nodes, we see that QMPR-2 scales better than MPR-1.

So from both cases, we conclude that QMPR-2 offers higher success ratio of packets transmission, and it scales well for high velocities within dense networks. These results confirm our hypothesis and reveal the positive effect of our proposal such as selected MPR are those nodes which maximize the utility function. Where each node publishes inside the TC messages all links with its MPRs which could constitute the paths from source to destination, then the set of MPRs forms a kind of backbone, in the mobile ad hoc network.

In Figure 7 we see that the average throughput per nodes behaves in the same way as the ratio of delivered packet. This comes to confirm the opposite dominance of our proposal that is explained by its ability of finding robust paths between destinations and sources in context of high mobility and dense network.

Figure 8 presents the average delay measured in packets transmission where there is a clear dominance of MPR-1. From the side of RQMPR, this bad performance is due to the increased number of MPR because of the features of the method used in the selection of relays. But this observation is valid until the number of nodes reaches 45 node; indeed, starting from this value, the delay of RQMPR performs better than MPR-1.

\section{Conclusion}

Generally, in proactive broadcasting scheme, the use of relays aims to reduce the broadcast messages senders and then the number of flooded messages. MPRs have been the core of broadcasting scheme and it is required to selected relays in reliable manner; indeed, our proposal is based on a multicriteria selection method of the latter node. Indeed, it is in a form of aggregated multicriteria problem, taking into account bandwidth, delay, and RTTQ. The innovative idea of the current work is to be aware of a set of criteria applied not just to the 1-hop neighbourhood but also to the 2-hop neighbourhood of a given node.

So to each of the criteria aforementioned, we have associated a metric and a technique of measurement, aiming to establish tradeoffs between each objective of those criteria. By summarizing the outcomes of our proposal, we conclude that it performs well in high mobility environment and scales better in dense network. Beside the performance that our approach realizes, namely, for PDR and throughput, some improvements still have been possible by using statistical techniques that allows correction of the new recorded values based on previous ones. Also we plan to create more composite metric using several techniques of measurements that have a direct impact on network performance such as residual energy of nodes. Further optimization can be done by monitoring the current network characteristics, based on some metrics, mapping these metrics to related routing parameters, and adjusting parameters if necessary. 
PDR

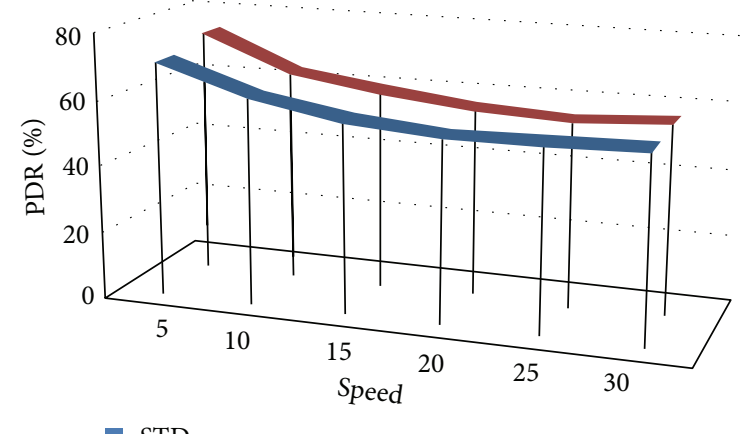

STD

RTTQ

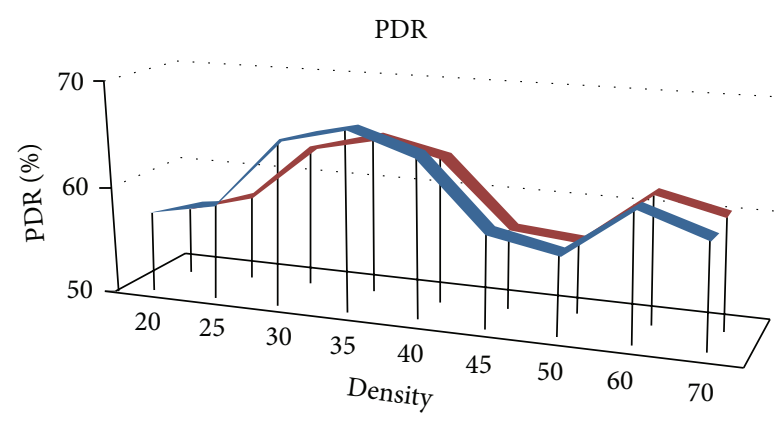

- STD

- RTTQ

(a)

(b)

FIGURE 6: (a) PDR varying velocity. (b) PDR varying density.

Throughput

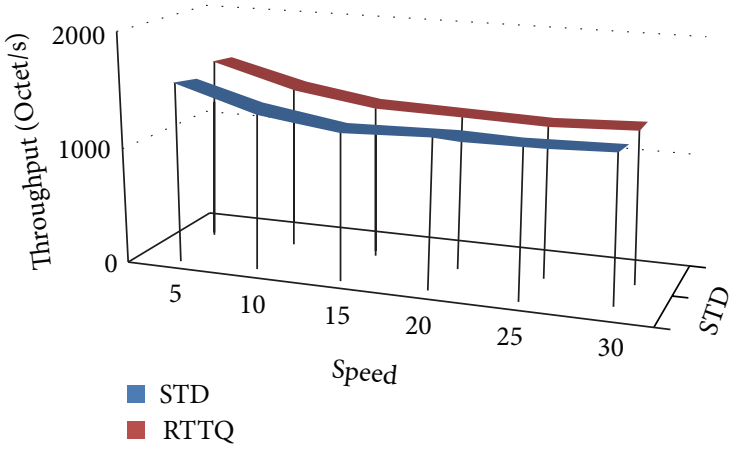

(a)

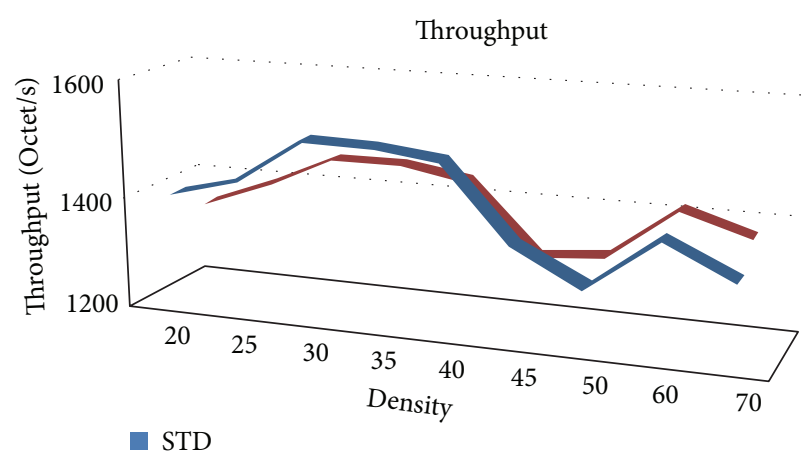

- RTTQ

Figure 7: (a) Throughput varying velocities. (b) Throughput varying number of nodes.

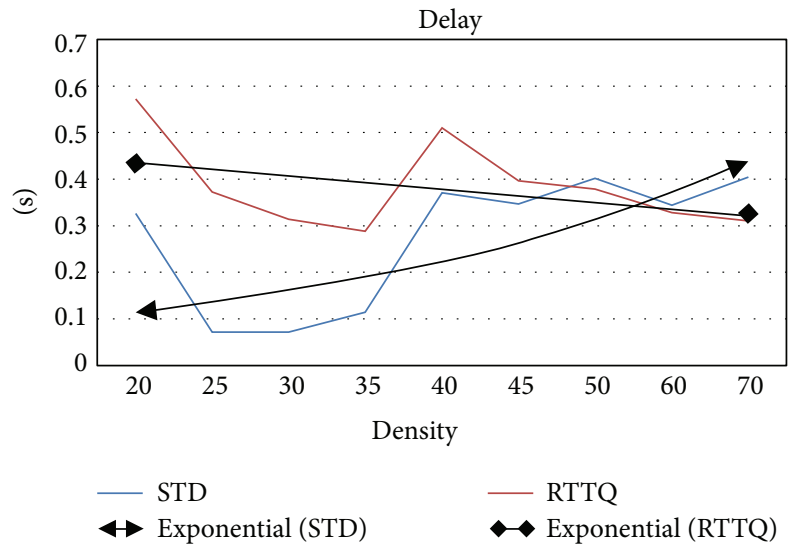

Figure 8: Delay measurement.

This adaptive process itself may also be dynamic, which means the node may receive feedback from the network and realise an interactive optimization.

\section{References}

[1] D. Nguyen and P. Minet, "Analysis of MPR selection in the OLSR protocol," in Proceedings of the 21st International Conference on Advanced Information Networking and ApplicationsWorkshops/Symposia (AINAW '07), pp. 887-892, May 2007.

[2] J. Leguay, V. Conan, and T. Friedman, "QoS routing in OLSR with several classes of service," in Proceedings of the 4th Annual IEEE International Conference on Pervasive Computing and Communications Workshops (PerCom Workshops '06), pp. 420425, March 2006.

[3] A. M. Poussard, W. Hamidouche, R. Vauzelle, Y. Pousset, and B. Parrein, "Realistic SISO and MIMO physical layer implemented in two routing protocols for vehicular ad hoc network," in Proceedings of the 9th International Conference on Intelligent Transport Systems Telecommunications (ITST '09), pp. 393-397, October 2009.

[4] H. Badis and K. A. Agha, "QOLSR, QoS routing for ad hoc wireless networks using OLSR," European Transactions on Telecommunications, vol. 16, no. 5, pp. 427-442, 2005.

[5] K. Yamada, T. Itokawa, T. Kitasuka, and M. Aritsugi, "Cooperative MPR selection to reduce topology control packets in OLSR," in Proceedings of the IEEE Region 10 Conference, TENCON 2010, pp. 293-298, November 2010.

[6] J. H. Ahn and T. J. Lee, "A multipoint relay selection method for reliable broadcast in ad hoc networks," in Proceedings of the International Conference on ICT Convergence (ICTC '11), pp. 616-617, September 2011. 
[7] R. Dube, C. D. Rais, K. Y. Wang, and S. K. Tripathi, "Signal stability-based adaptive routing (SSA) for ad hoc mobile networks," IEEE Personal Communications, vol. 4, no. 1, pp. 36-45, 1997.

[8] T. Goff, N. B. Abu-Ghazaleh, D. S. Phatak, and R. Kahvecioglu, "Preemptive routing in ad hoc networks," in Proceedings of the 7th Annual International Conference on Mobile Computing and Networking, pp. 43-52, Rome, Italy, July 2001.

[9] D. Djenouri and I. Balasingham, "LOCALMOR: LOCALized Multi-Objective Routing for wireless sensor networks," in Proceedings of the IEEE 20th Personal, Indoor and Mobile Radio Communications Symposium (PIMRC '09), pp. 1188-1192, September 2009.

[10] D. Johnson and G. Hancke, "Comparison of two routing metrics in OLSR on a grid based mesh network," Ad Hoc Networks, vol. 7, no. 2, pp. 374-387, 2009.

[11] Y. Fu, X. Wang, and S. Li, "Performance comparison and analysis of routing strategies in mobile ad hoc network," in Proceedings of the International Conference on Computer Science and Software Engineering (CSSE '08), pp. 505-510, December 2008.

[12] J. Toutouh, J. Garcia-Nieto, and E. Alba, "Intelligent OLSR routing protocol optimization for VANETs," IEEE Transactions on Vehicular Technology, vol. 61, pp. 1884-1894, 2012.

[13] C. Gomez, D. Garcia, and J. Paradells, "Improving performance of a real ad-hoc network by tuning OLSR parameters," in Proceedings of the 10th IEEE Symposium on Computers and Communications (ISCC '05), pp. 16-21, June 2005.

[14] K. Ghada, J. Li, and Y. Ji, "Cross-layer design for topology control and routing in MANETs," Wireless Communications and Mobile Computing, vol. 12, no. 3, pp. 257-267, 2012.

[15] S. Marwaha, D. Srinivasan, C. K. Tham, and A. Vasilakos, "Evolutionary fuzzy multi-objective routing for wireless mobile ad hoc networks," in Proceedings of the 2004 Congress on Evolutionary Computation (CEC '04), vol. 2, pp. 1964-1971, June 2004.

[16] W. Su, S. J. Lee, and M. Gerla, "Mobility prediction and routing in ad hoc wireless networks," International Journal of Network Management, vol. 11, pp. 3-30, 2001.

[17] W. Su and M. Gerla, "IPv6 flow handoff in ad hoc wireless networks using mobility prediction," in Proceedings of the IEEE Global Telecommunication Conference (GLOBECOM '99), vol. 1, pp. 271-275, December 1999.

[18] D. B. Johnson, D. A. Maltz, and J. Broch, "DSR: the dynamic source routing protocol for multihop wireless ad hoc networks," in Ad Hoc Networking, pp. 139-172, Addison-Wesley Longman Publishing, 2001.

[19] N. Meghanathan, "Exploring the stability-energy consumptiondelay-network lifetime tradeoff of mobile ad hoc network routing protocols," Journal of Networks, vol. 3, no. 2, pp. 17-28, 2008.

[20] X. M. Zhang, F. F. Zou, E. B. Wang, and D. K. Sung, "Exploring the dynamic nature of mobile nodes for predicting route lifetime in mobile Ad hoc networks," IEEE Transactions on Vehicular Technology, vol. 59, no. 3, pp. 1567-1572, 2010.

[21] S. Jiang, "An enhanced prediction-based link availability estimation for MANETs," IEEE Transactions on Communications, vol. 52, no. 2, pp. 183-186, 2004.

[22] S. Jiang, D. He, and J. Rao, "A prediction-based link availability estimation for routing metrics in MANETs," IEEE/ACM Transactions on Networking, vol. 13, no. 6, pp. 1302-1312, 2005.
[23] Q. Han, Y. Bai, L. Gong, and W. Wu, "Link availability prediction-based reliable routing for mobile ad hoc networks," IET Communications, vol. 5, no. 16, pp. 2291-2300, 2011.

[24] T. Camp, J. Boleng, and V. Davies, "A survey of mobility models for ad hoc network research," Wireless Communications and Mobile Computing, vol. 2, no. 5, pp. 483-502, 2002.

[25] F. Bai, N. Sadagopan, and A. Helmy, "The IMPORTANT framework for analyzing the impact of mobility on performance of RouTing protocols for Adhoc NeTworks," Ad Hoc Networks, vol. 1, no. 4, pp. 383-403, 2003.

[26] N. Sadagopan, F. Bai, B. Krishnamachari, and A. Helmy, "PATHS: analysis of PATH duration statistics and their impact on reactive MANET routing protocols," in Proceedings of the $4 \mathrm{~h}$ ACM International Symposium on Mobile Ad Hoc Networking and Computing, pp. 245-256, Annapolis, Md, USA, June 2003.

[27] J. Broch, D. A. Maltz, D. B. Johnson, Y. C. Hu, and J. Jetcheva, "A performance comparison of multi-hop wireless ad hoc network routing protocols," in Proceedings of the 4th Annual ACM/IEEE International Conference on Mobile Computing and Networking, Dallas, Tex, USA, 1998.

[28] S. Mo, J. Hsu, J. Gu, M. Luo, and R. Ghanadan, "Network synchronization for distributed MANET," in Proceedings of the IEEE Military Communications Conference (MILCOM '08), pp. 1-7, November 2008.

[29] A. Ouacha, N. Lakki, B. Bouamoud, A. Habbani, J. E. ABBADI, and M. Elkoutbi, "Reliable MPR selection based on link lifetime-prediction method," in Proceedings of the 10th IEEE International Conference on Networking, Sensing and Control (ICNSC '13), pp. 11-16, Paris-Evry University, Evry, France, April 2013.

[30] H. Badis, A. Munaretto, K. Al Agha, and G. Pujolle, "Optimal path selection in a link state QoS routing protocol," in Proceedings of the IEEE 59th Vehicular Technology Conference (VTC '04), vol. 5, pp. 2570-2574, May 2004.

[31] Y. Ge, T. Kunz, and L. Lamont, "Quality of service routing in AdHoc networks using OLSR," in Proceedings of the 36th Annual Hawaii International Conference on System Sciences (HICSS '03), vol. 9, 2003, Track 9.

[32] O. Liang, Y. A. Sekercioglu, and N. Mani, "A survey of multipoint relay based broadcast schemes in wireless ad hoc networks," Communications Surveys \& Tutorials, vol. 8, pp. 3046, 2006.

[33] H. Alwan and A. Agarwal, "Multi-objective reliable multipath routing for wireless sensor networks," in Proceedings of the IEEE Globecom Workshops (GC '10), pp. 1227-1231, December 2010.

[34] R. L. Gomes, W. A. Moreira, J. J. H. Ferreira, and A. J. G. Abele, "LatinCon14-providing QoE and QoS in wireless mesh networks through dynamic choice of routing metrics," IEEE Latin America Transactions, vol. 8, no. 4, pp. 454-462, 2010.

[35] G. Koloniari and E. Pitoura, "A game-theoretic approach to the formation of clustered overlay networks," IEEE Transactions on Parallel and Distributed Systems, vol. 23, no. 4, pp. 589-597, 2012.

[36] D. Mahjoub and H. El-Rewini, "Adaptive constraint-based multi-objective routing for wireless sensor networks," in Proceedings of the IEEE International Conference on Pervasive Services (ICPS '07), pp. 72-75, July 2007.

[37] S. Corson and J. Macker, Routing Protocol Performance Issues and Evaluation Considerations, RFC 2501, Mobile ad hoc Networking (MANET), 1999. 

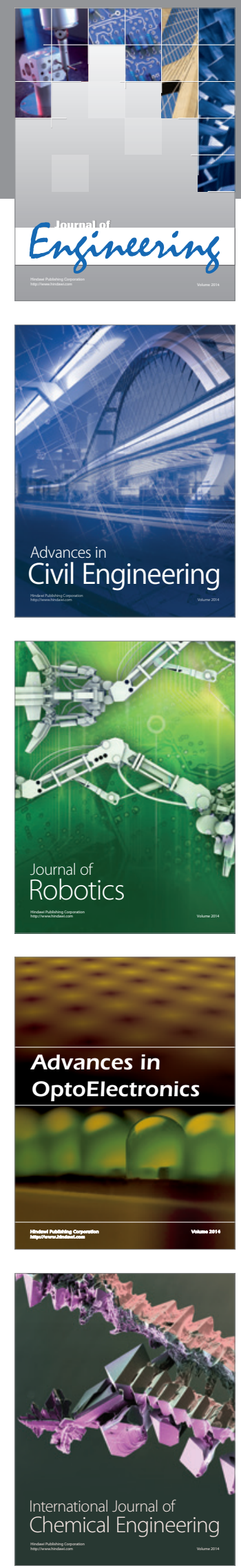

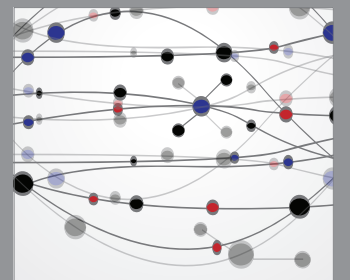

The Scientific World Journal
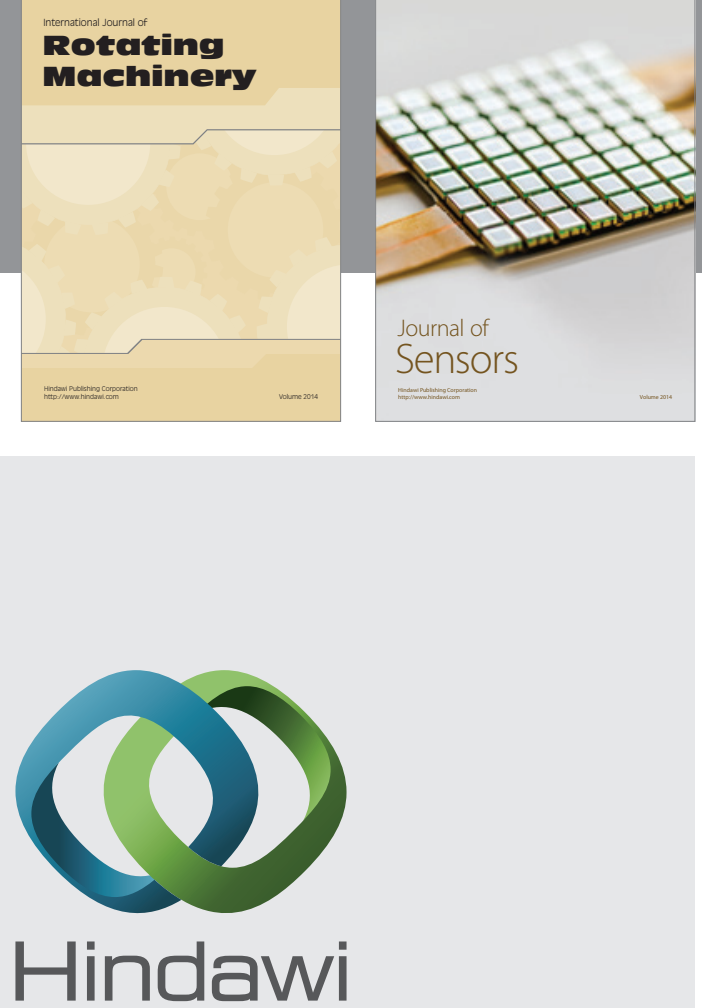

Submit your manuscripts at http://www.hindawi.com
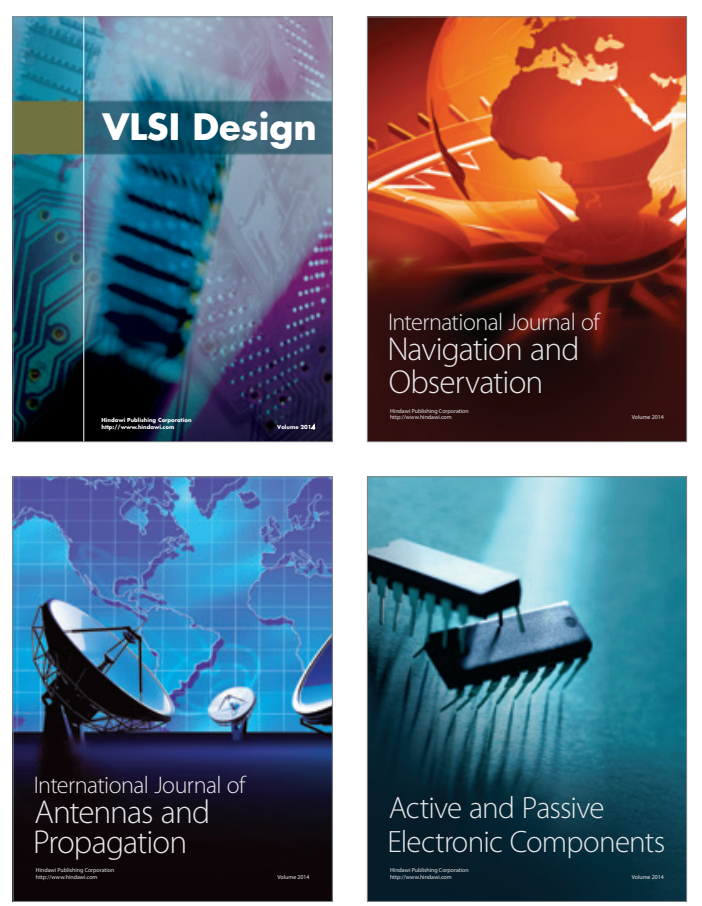
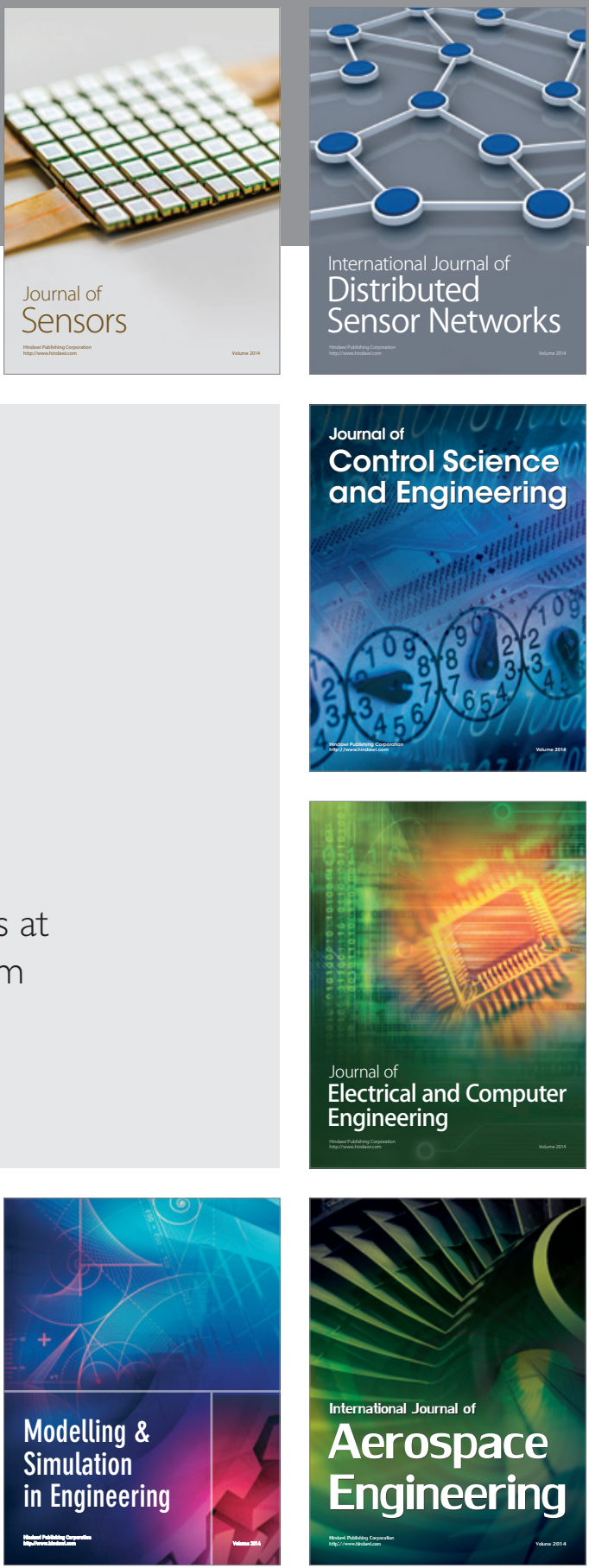

Journal of

Control Science

and Engineering
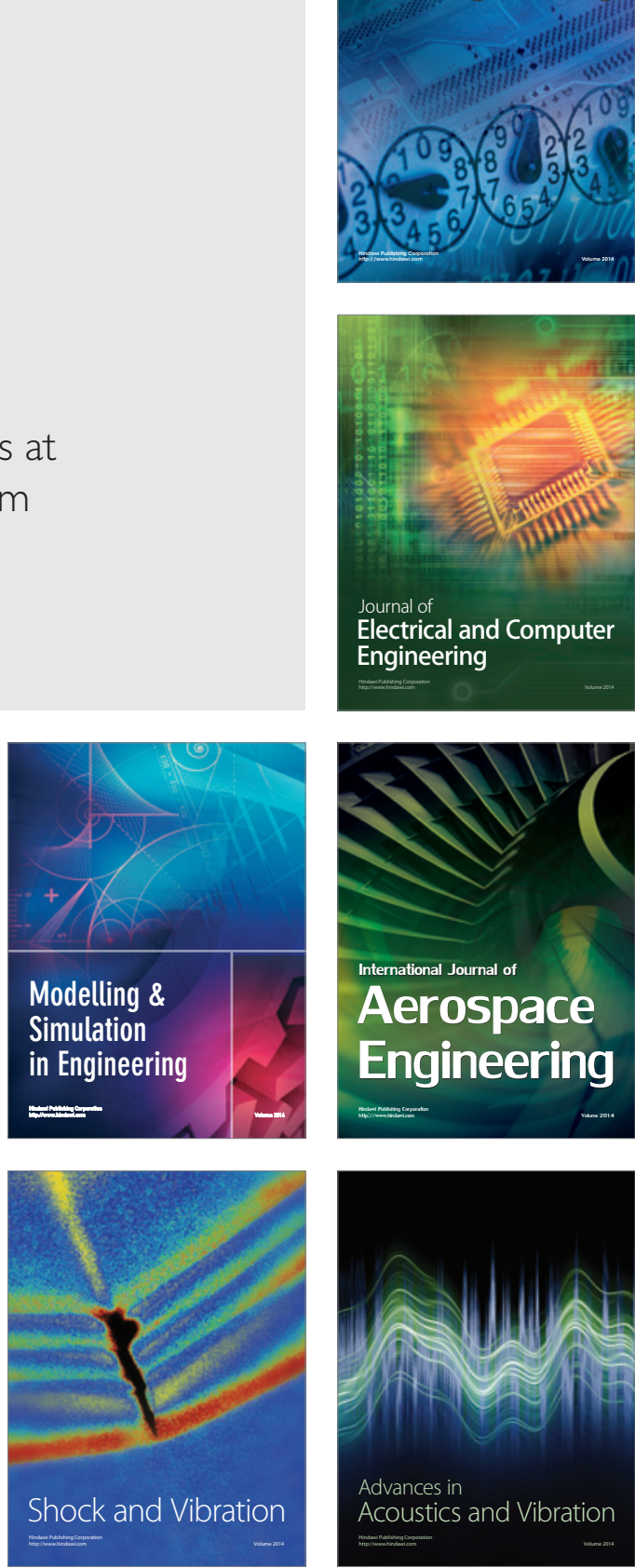